\title{
Threshold Criterion for a Space Simulation Beam-Plasma Discharge
}

\author{
E. P. Szuszczewicz, ${ }^{1}$ K. Papadopoulos, ${ }^{2}$ W. Bernstein, ${ }^{3}$ C. S. Lin, ${ }^{4}$ \\ AND D. N. WALKER ${ }^{1}$
}

\begin{abstract}
We have conducted an experimental and theoretical study of the threshold characteristics of a space simulation beam-plasma discharge with emphasis on density profiles and a density-dependent ignition criterion. The study included various beam-plasma conditions covering beam currents from 8 to $85 \mathrm{ma}$, beam energies from 0.8 to $2.0 \mathrm{keV}$, and magnetic fields at 0.9 and $1.5 \mathrm{G}$. The study included experimental determinations of radial profiles of electron density for each of the selected conditions extending from a low-density, pre-beam-plasma discharge state to a strong beam-plasma discharge condition. At beam-plasma discharge threshold it was determined that $\left\langle\omega_{p} / \omega_{c}\right\rangle=5.4$ was the densitydependent ignition criterion. The experimental results are shown to agree with detailed model calculations, which consider the beam-plasma discharge to be produced by large-amplitude electron plasma waves resulting from the beam-plasma interaction.
\end{abstract}

\section{INTRODUCTION}

A cold electron beam propagating through a weakly ionized plasma will, under proper conditions, produce a modified beam-plasma state known as the beam-plasma discharge (BPD). This discharge state has received considerable attention in recent years as a result of increased interest in mechanisms for vehicle neutralization during spaceborne accelerator experiments [Bernstein et al., 1980; Cambou et al., 1978; Galeev et al., 1976], enhanced beam-plasma ionization processes [Bernstein et al., 1978], and, in general, single-particle or collective phenomena initiated by beams injected into neutral gas and charged-particle environments [Hess et al., 1971; Winckler et al., 1975; Hendrickson and Winckler, 1976; Cambou et al., 1975; Monson and Kellogg, 1978; Szuszczewicz et al., 1979; Jost et al., 1980; Winckler, 1980].

The BPD appears at a critical energetic-electron-beam current $I_{B}{ }^{c}$, with the transition from single-particle behavior $\left(I_{B}<I_{B}{ }^{c}\right.$, pre-BPD) to collective processes $\left(I_{B}>I_{B}{ }^{c}\right.$, solid BPD) described as follows for conditions in which the plasma is created by the beam itself.

1. As an electron beam linearly interacts with a neutral gas, it collisionally produces a plasma with a density that varies directly with the magnitude of the beam current for a fixed beam energy.

2. As the beam current is increased to a critical value $I_{B}{ }^{c}$, a two-stream instability sets in and the electric fields of the excited waves heat the electrons to energies comparable to the ionization energy. The 'heated' electrons create an enhanced ionization process, which results in an avalanche breakdown, the BPD.

Bernstein et al. [1979] have reported the dependence of this critical current $I_{B}{ }^{c}$ on various experimental parameters

\footnotetext{
${ }^{1}$ Ionospheric Diagnostics Section, E. O. Hulburt Center for Space Research, Space Science Division, Naval Research Laboratory, Washington, D. C. 20375.

${ }^{2}$ Science Applications, Inc., McLean, Virginia 22102.

${ }^{3}$ Center for Space Physics, Department of Space Physics and Astronomy, Rice University, Houston, Texas 77001.

${ }^{4}$ Bendix Field Engineering Corporation, Columbia, Maryland 21045.

This paper is not subject to U.S. copyright. Published in 1982 by the American Geophysical Union.
}

as

$$
I_{B}{ }^{c} \propto \frac{V_{B}^{3 / 2}}{B^{0.7} P L}
$$

where $V_{B}, B, P$, and $L$ are the beam energy (voltage), the superimposed magnetic field, the ambient neutral pressure, and the beam length (gun-to-collector distance), respectively. While the $I_{B}^{c}=I_{B}^{c}\left(V_{B}, B, P, L\right)$ relationship was established among the controlling system parameters (with the $I_{B}{ }^{c} \propto P^{-1}$ behavior applicable at $P \leqslant 10^{-5}$ torr), a clear dependence on plasma density was expected. Early thoughts suggested that $\omega_{p} \geq \omega_{c}$ satisfied ignition threshold criteria [Bernstein et al., 1979; Getty and Smullin, 1963]. We have since had the opportunity to test this idea under various beam-plasma conditions. Our results include (1) time-delay data and associated analyses indicating that BPD ignition does indeed occur at a critical density, (2) direct measurements of plasma density near ignition threshold with determinations of $\omega_{p} / \omega_{c}$, and (3) a theoretical analysis that predicts the critical density criterion.

In subsequent sections we present the experimental and theoretical details, which establish the density-dependent threshold conditions for BPD at $3.5 \leqslant \omega_{p} / \omega_{c} \leqslant 6.9$.

\section{EXPERIMENT CONFIGURATION AND RESULTS}

The experiment was conducted in a large vacuum chamber facility at the NASA Johnson Space Center with an experimental configuration similar to those employed in earlier investigations [Bernstein et al., 1979]. The configuration is illustrated in Figure 1 with specific emphasis on the pulsed plasma probe measurement and the associated procedure for $\omega_{p} / \omega_{c}$ determinations. Not shown are the previously described diagnostics [Bernstein et al., 1979; Jost et al., 1980] including the 3914- $\AA$ scanning photometer, the segmented current collector, the energetic electron electrostatic analyzer, and the remote wave detection antenna and spectrum analyzer system. The beam was generated by a tungstencathode Pierce-type diode gun, mounted on a positioncontrolled cart. In all cases the beam was injected parallel to the magnetic field $\bar{B}$ and terminated on a $3 \times 3 \mathrm{~m}$ target suspended about $20 \mathrm{~m}$ above the gun aperture. A combination of coil current and the earth's magnetic field established the $B$ field at selected levels up to $1.5 \mathrm{G}$.

In most cases the beam was injected into a neutral gas with no pre-beam plasma; however, the experimental investiga- 


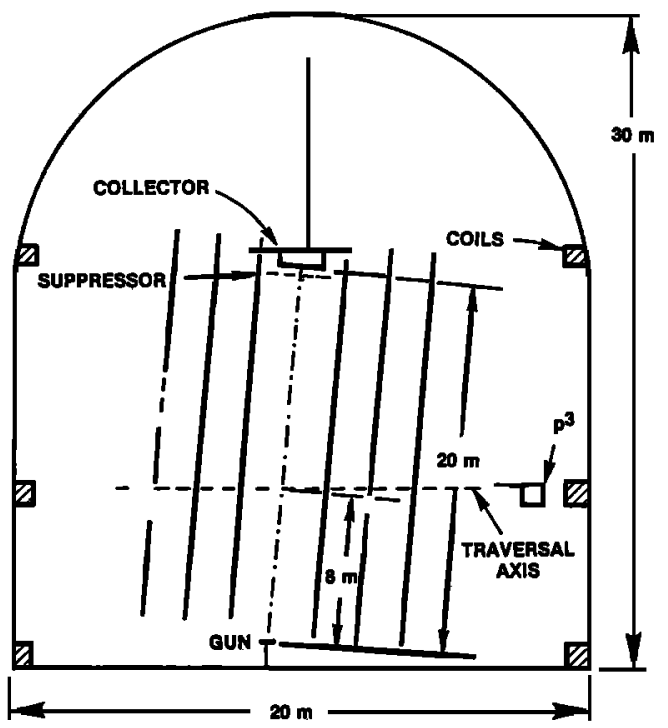

Fig. 1. Experiment configuration.

tion included two cases in which the chamber was filled with a plasma created by a Kauffman-type argon ion thruster. In these cases the pre-beam plasma density was lower than the critical density at BPD ignition.

The investigation was conducted in two stages. First, pulsed-gun experiments were carried out to test the concept of a density-dependent threshold condition against the original ideas of Getty and Smullin [1963]. After the concepts check, a series of direct density measurements was conducted to quantitatively establish the density criteria. We describe the results in the order in which the experiments were conducted.

\section{Time Delay Arguments for $N_{e}^{c}$}

In pulsed-beam experiments with $I_{B} \gg I_{B}{ }^{c}$, Getty and Smullin [1963] identified three sequential phases in the temporal evolution into the BPD: (1) a quiescent stable period, (2) an intermediate period during which the gross features of the beam remained unchanged but waves with $f \approx$ $f_{c e}$ were present, and (3) BPD ignition. They suggested that phases 1 and 2 corresponded to the time required for buildup of the ambient density to a critical value . . . a value at which the BPD ignited. To test this concept in the space simulation beam-plasma efforts at the JSC facility a series of pulsedbeam measurements were performed to study the temporal evolution of the BPD in the configuration shown in Figure 1. All diagnostics sensitive to the transition to BPD demonstrated identical time delays between beam current initiation and BPD ignition. At low pressure $\left(<4\left(10^{-6}\right)\right.$ torr) the Getty and Smullin phase 2 (characterized by $f \approx f_{c e}$ waves) was clearly evident. Figure 2 shows the measured delay times as a function of beam current with values ranging from 0.1 to 20 ms. The results in Figure 2 can be understood in terms of a simple time dependent model. If it is assumed that the plasma loss rate is proportional to its density, then the temporal buildup of plasma by electron-neutral collisions is given by

$$
\frac{d N_{e}}{d t}=\gamma-\nu N_{e}
$$

Equation (2) has the solution

$$
N_{e}(t)=\frac{\gamma}{\nu}\left(1-e^{-\nu t}\right)
$$

where $\gamma=I_{B} \sigma N_{0} / e A$ is the electron-ion pair production rate, $\nu$ is the loss rate, $\sigma$ is the electron impact ionization cross section, $N_{0}$ is the neutral density, and $A$ is the beam cross section. At short times, the density will increase linearly with time $\left(N_{e}=\gamma t\right)$, and at longer times the density will approach the time independent value $\dot{N}_{e}=\gamma / \nu$. If, indeed, there is a critical density $N_{e}^{c}$ required for BPD ignition and if we define its relationship to a critical beam current by $N_{e}^{c}=$ $\left(\sigma N_{0} / e A \nu\right) I_{B}{ }^{c}$, then the time delay $t_{d}$ until $N_{e}(t)=N_{e}^{c}$ can be obtained from (3) and written as

$$
t_{d}=-\frac{1}{\nu} \ln \left(1-\frac{I_{B}{ }^{c}}{I_{B}}\right)
$$

For $I_{B} \approx I_{B}{ }^{c}$, relatively long delay times should be observed with a rapid decrease in $t_{d}$ as $I_{B}$ is increased. This is exactly the behavior demonstrated in Figure 2, where the solid curve has been normalized to the data by selecting $I_{B}{ }^{c}=20 \mathrm{ma}$ and $\nu \approx 400 \mathrm{~s}^{-1}$. The good agreement between the simple model calculations and the experimental results supports the concept of a critical density threshold for BPD ignition, but the normalization procedure provides little measure for an absolute value of $N_{e}^{c}$. Specific determinations of the critical density threshold criteria are detailed in the next two sections.

\section{Direct Measurements of Critical Density}

To establish quantitatively the density dependent threshold criterion for the ignition of a space simulation BPD, emphasis was placed on the direct measurement of plasma density profiles over a range of beam-plasma conditions covering beam currents from 8 to $85 \mathrm{ma}$, beam energies from 0.8 to $2.0 \mathrm{keV}$, and magnetic fields at 0.9 and $1.5 \mathrm{G}$. The

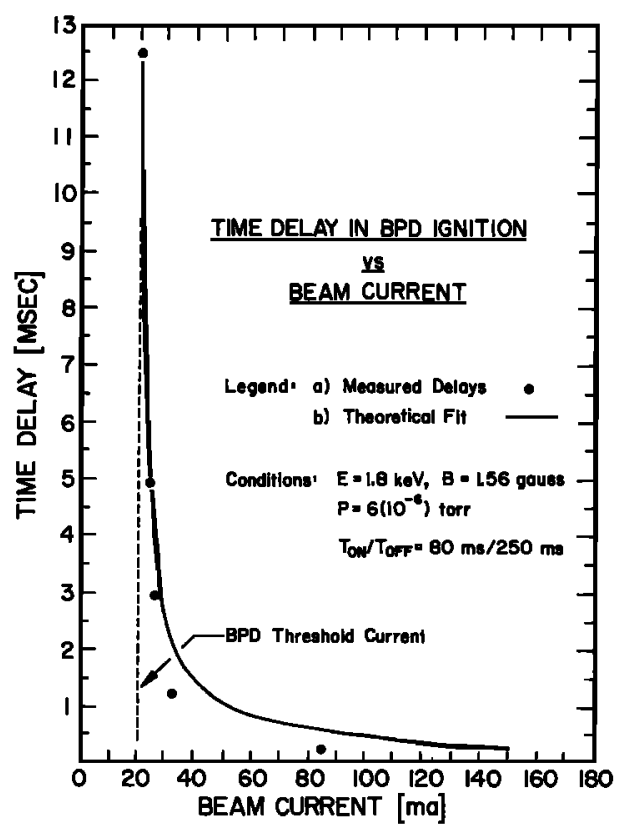

Fig. 2. Time delay in the ignition of the beam-plasma discharge with threshold current at 20 ma. Electron gun operation was in the pulsed mode with $T_{\text {on }} / T_{\text {off }}=80 \mathrm{~ms} / 250 \mathrm{~ms}$. 


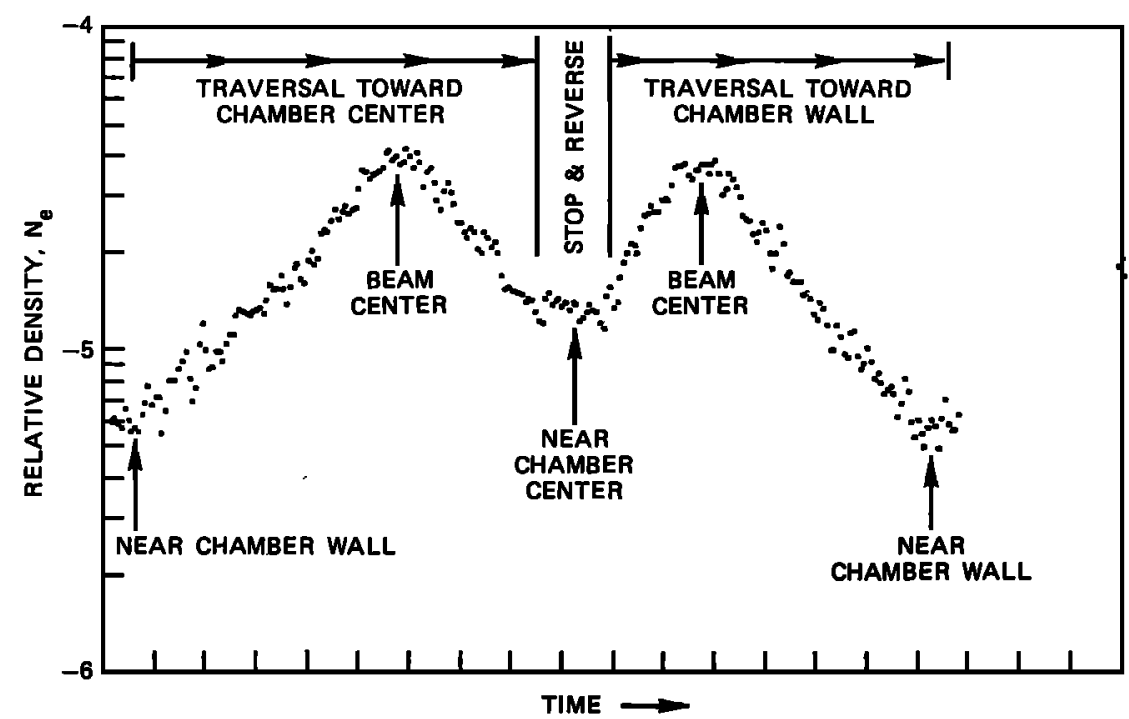

Fig. 3. Radial profile of relative electron density under pre-BPD conditions. Run $57\left(I_{B}, V_{B}, B\right)=(7 \mathrm{ma}, 1.3 \mathrm{keV}$, $0.9 \mathrm{G}$ ). The figure shows two cuts through the beam-plasma profile; as time increases from left to right the plasma density probe moves into and through the beam center, then reverses and passes through the beam a second time. The symmetry verifies that beam-plasma conditions were stable during the execution of the radial traversal.

procedure involved experimental determinations of radial profiles of electron density for each of the selected conditions extending from a low-density, pre-BPD state to a strong BPD condition. The experimental configuration, illustrated in Figure 1, involved a pair of pulsed-plasma-probes $\left(\mathrm{P}^{3}\right)$ mounted on a radial traversal mechanism positioned at approximately $8 \mathrm{~m}$ above the injection point of the beam. Each of the probes provided simultaneous measurements of electron density $N_{e}$, temperature $T_{e}$, plasma potential $V_{\infty}$, and density fluctuation power spectra $\delta N_{e}\left(\rightarrow P_{n}(k)\right)$ with capabilities for the associated diagnostics in a dynamic plasma environment and under test conditions that could contaminate electrode surfaces [Holmes and Szuszczewicz, 1975, 1981; Szuszczewicz and Holmes, 1975, 1976].

The plasma density measurements were made for seven different conditions, each identified by preselected values for $V_{B}, B, P$ and the existence or nonexistence of a pre-beam plasma. For each condition a steady state value for $\boldsymbol{I}_{B}$ was set, the traversal mechanism was exercised, and an electron density profile was recorded. A sample profile collected under pre-BPD conditions is presented in Figure 3. The abscissa is time relative to the start of the radial traversal, and the ordinate is relative electron density as determined by base line electron-saturation currents collected by the $\mathrm{E}$ probe. (The second in the two-probe configuration was defined as the I probe because the associated base line currents were collected in the ion-saturation portion of the probe's current-voltage characteristic [Holmes and Szuszczewicz, 1975, 1981].) At the start of each traversal the probe was at its outermost position relative to the center of the chamber. As time increased, the probe was moved into and through the beam; at maximum penetration toward the chamber center, the traversal system was reversed, allowing a second measurement of the density profile as the probe moved back to its original outermost position. With this procedure the probe's maximum penetration toward the chamber center is identified by the symmetry point in the 'double' profile. The symmetry in the double profile provid- ed confidence that beam-plasma conditions were unchanged during the measurement.

Absolute electron densities were determined by standard $\mathrm{P}^{3}$ analysis procedures summarized graphically in Figure 4. The technique provides a high-frequency $\left(10^{3} \mathrm{~Hz}\right.$ in this experiment) determination of relative electron density through the direct measurement of baseline electron-saturation currents. (The baseline currents provide the fundamental data for the relative density profiles presented in Figures $3,5 a$, and $5 b$.) Simultaneously, the technique generates a 'conventional' Langmuir probe characteristic. The relative density fluctuations (as indicated by the variations in the base line current) are then unfolded from the raw, uncorrected probe characteristic (Figure $4 a$ ) yielding a smooth, corrected curve (Figure $4 b$ ) to which conventional $N_{e}$ analysis procedures [Chen, 1965; Szuszczewicz and Holmes, 1977] are applied. This procedure, utilized for all beam-plasma conditions included in this investigation, provided a direct scaling of baseline current measurements to absolute electron densities.

Complete profile information and associated plasma wave signatures are presented for two independent conditions in Figures 5 and 6. (Note: Because of changes in plasma potential, the relative density profiles do not maintain the same scaling to absolute values from run to run.) For values of $\left(V_{B}, B\right)=(1.3 \mathrm{keV}, 0.9 \mathrm{G})$ and $(2.0 \mathrm{keV}, 1.5 \mathrm{G})$ in Figures 5 and 6 , respectively, the beam current $I_{B}$ was stepped through a sequence allowing for complete coverage of conditions that encompassed pre-BPD, threshold-BDP, and solidBPD. Because $I_{B}{ }^{c}=I_{B}\left(V_{B}, B, P, L\right)$ is known to exhibit hysteresis as $I_{B}$ is varied about the critical value $I_{B}{ }^{c}$, the following procedure was utilized in the step-wise selection of beam current levels.

1. With $V_{B}$ fixed, $I_{B}$ was steadily increased in a 'search' mode to determine an approximate value for $I_{B}{ }^{c}$.

2. $I_{B}$ was reset to zero, then increased slowly to $I_{B}{ }^{c}$ with careful observation of the RF spectrum. Threshold was defined as that level at which the RF spectrum was in 

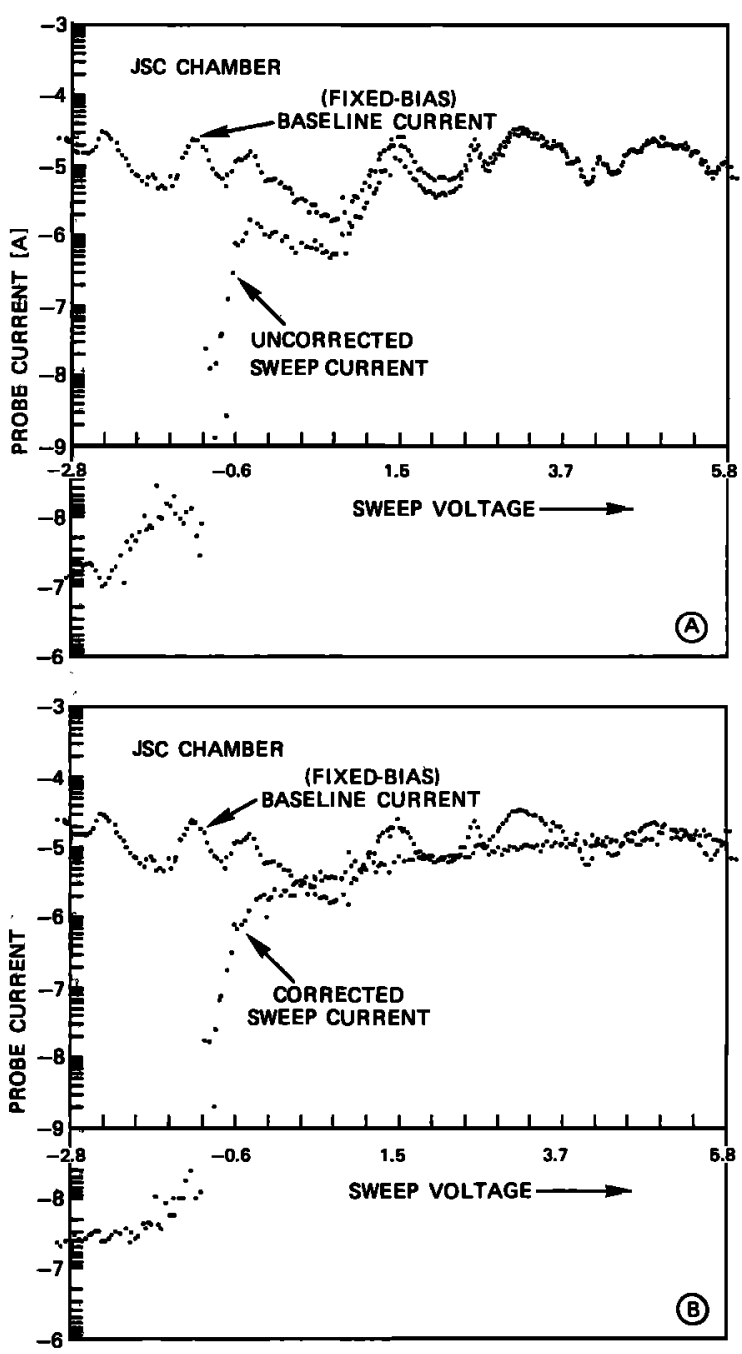

Fig. 4. (a) Sample of raw probe data showing the effects of density fluctuations (baseline electron-saturation currents) on the probe's current-voltage characteristic (sweep currents). (b) The 'corrected' characteristic that results when density fluctuations have been unfolded. The electron saturation portion of the characteristic is then amenable to conventional analyses for determination of $N_{e}$ (see text).

transition from its pre-BPD signature (flat spectrum at high frequencies, $f \geq f_{p}$ ) to its solid BPD characteristic (intense features at $f \gtrsim f_{p}$ ). At this transition level (defined here as threshold) the beam-plasma system oscillated between its two states at a repetition frequency generally observed to be no greater than several hertz. If the RF spectrum locked up in à chàracteristically stable RF BPD signature, step 2 was restarted and the previous $I_{B}{ }^{c}$ level more carefully approached. (It is important to note that the time dependent photographs of the threshold RF spectrum, e.g., runs 57 and 81 , erroneously indicate a solid BPD spectrum and do not reflect the oscillations between the two beam-plasma states.) The conditions at threshold and under BPD are summarized in Table 1 where the peak density $N_{e}^{\max }$, associated plasma frequency $2 \pi \omega_{p}{ }^{\max }$, and plasma-to-cyclotron frequency ratio $\omega_{p}{ }^{\text {max }} / \omega_{c}$ are also listed. The results can be summarized by $\omega_{p} / \omega_{c}=5.4_{-1.9^{+1.3}}$ as the density dependent threshold condition for the BPD. As we will show in the next section, this result is consistent with a threshold model, which assumes that BPD is triggered by the onset of a beam plasma instability.

It is proper to introduce one qualification regarding the experimentally determined values for $\omega_{p} / \omega_{c}$. By virtue of the experimental procedure they could represent averages between two beam-plasma states (pre-BPD and solid BPD). The consequence of this in the interpretation of the experimental values is merely one of definition. For example, if threshold were strictly defined as the maximum density under stable pre-BPD conditions (i.e., no oscillations into the solid BPD state), then $\left\langle\omega_{p} / \omega_{c}\right\rangle=5.4$ would represent an upper limit. Extrapolation of complementary sets of data suggests that the associated 'adjustment' in critical density for cases at $0.9 \mathrm{G}$ is inconsequential, while corresponding adjustments at $1.5 \mathrm{G}$ could be as large as a factor of $2-3$. The end results would be $\omega_{p} / \omega_{c} \approx 4.4$ as compared with the experimentally defined average at 5.4 .

Before proceeding to the theoretical treatment, it is appropriate to discuss several of the plasma density and waverelated features in the data presented in Figures 5 and 6.

Run 56 (upper left panel Figures $5 a$ and $5 b$ ) is very typical of a pre-BPD environment, in that the beam center (see, e.g., Figure 3 ) is well defined and of relatively narrow cross section, with an RF signature (upper left panel Figure $5 b$ ) that is flat in character. At BPD threshold the beam-plasma system intermittently emits RF at and around the plasma frequency, and the plasma expands in cross section (lower left panel, Figure $5 a$ ) eliminating the narrow beam profile characteristic of the pre-BPD state (Figures 3 and $5 a$ upper left). We note that the RF signatures are highly time dependent, generally fluctuating in intensity and in frequency. Similarly, the local plasma densities are highly time dependent (see, e.g., $I_{B}$ fluctuations in Figure 4) with that dependency averaged out over $1-s$ intervals for the relative density profiles in Figure 5 and 6.

As $I_{B}$ is increased above the threshold level (runs 58 and 59 in Figures $5 a$ and $5 b$ ), the plasma density increases and the radial profiles take on variable configurations. In run 58, for example, the beam center is 'depleted' with an increased density level at its edges. This configuration has been observed in a number of cases and is thought to manifest electron heating in the beam core with generally higher diffusion rates and associated losses. The profile becomes more complex in run 59 , possibly due to higher order diffusion modes.

The results in Figure 6 have been selected to present information at a higher applied magnetic field and to illustrate that the description of beam-plasma profiles offered in connection with Figure 5 is by no means considered universally applicable to the various levels of the BPD. O'ur current level of understanding does not allow for a detailed causeeffect description of each and every profile, but at present we would point to Figure $5 a$ as the simplest configuration that illustrates the transition from pre-BPD to the solid BPD state.

\section{Theoretical Considerations and Discussion OF RESUlts}

Before presenting the theoretical details of the BPD threshold criteria as applied to the present experiment, it is important to discuss some aspects of our model that play a key role in the interpretation of the experimental results. 
For all experimental parameters the relevant mean free paths (i.e., thermalization, ionization, etc.) were much longer than the system length $L$. This, as discussed in detail by Papadopoulos [1981] for a finite system, leads to a requirement for axial confinement of both the ionizing and the ambient electrons. As shown in the above paper, the required axial confinement can be achieved if the energy deposited by the beam electrons is absorbed by only a few ambient electrons (i.e., 1\%), which are accelerated to energies larger than the ionization potential. A fraction of these electrons quickly escapes the system, thereby building potential sheaths at the ends. The sheaths accelerate ambient ions to escape at the rate of the fast electrons, while trapping the cold part of the energetic electron population. A detailed description of the process can be found in the work of Papadopoulos [1981]. For the purposes of the present paper, we retain the requirement that the collisionless mechanism responsible for BPD should deposit most of its energy in suprathermal tails with energy much larger than the ionization energy while leaving the majority of the electrons cold. As discussed in many publications [Papadopoulos and Coffey, 1974a, $b$; Papadopoulos and Rowland, 1978; Linson and Papadopoulos, 1980; Papadopoulos, 1982], this can be achieved if the frequency of the excited waves is near the
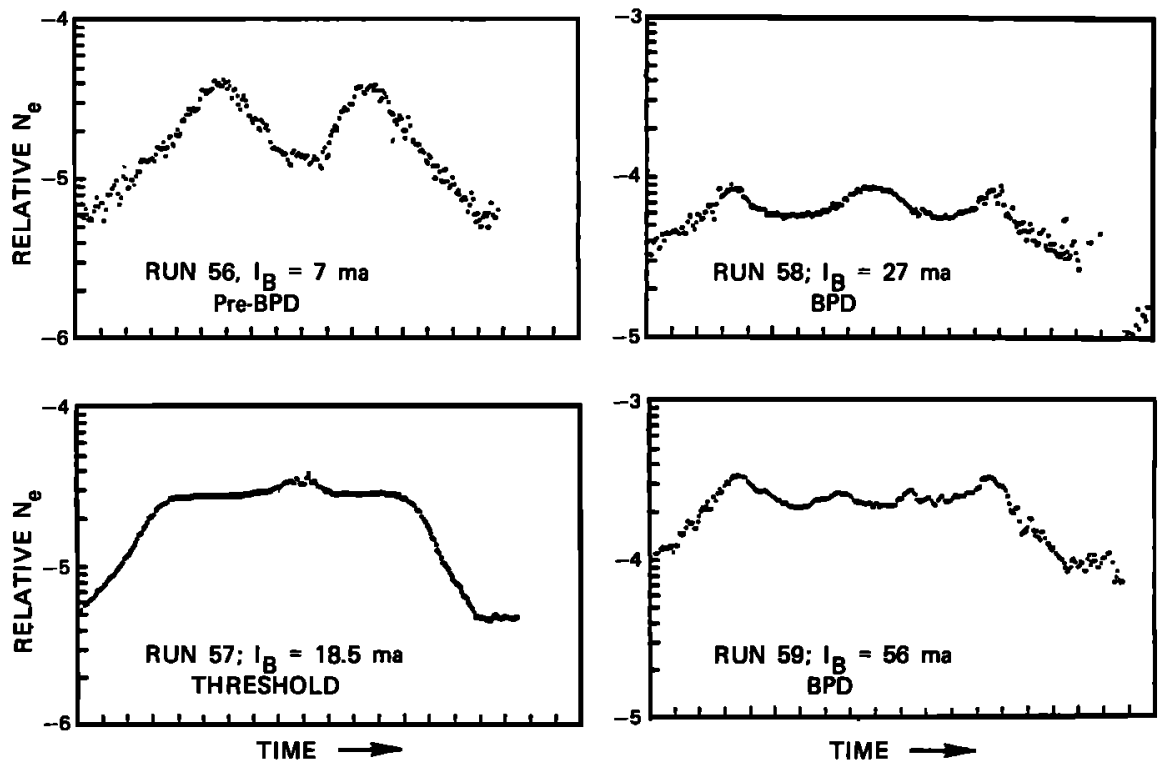

Fig. $5 a$
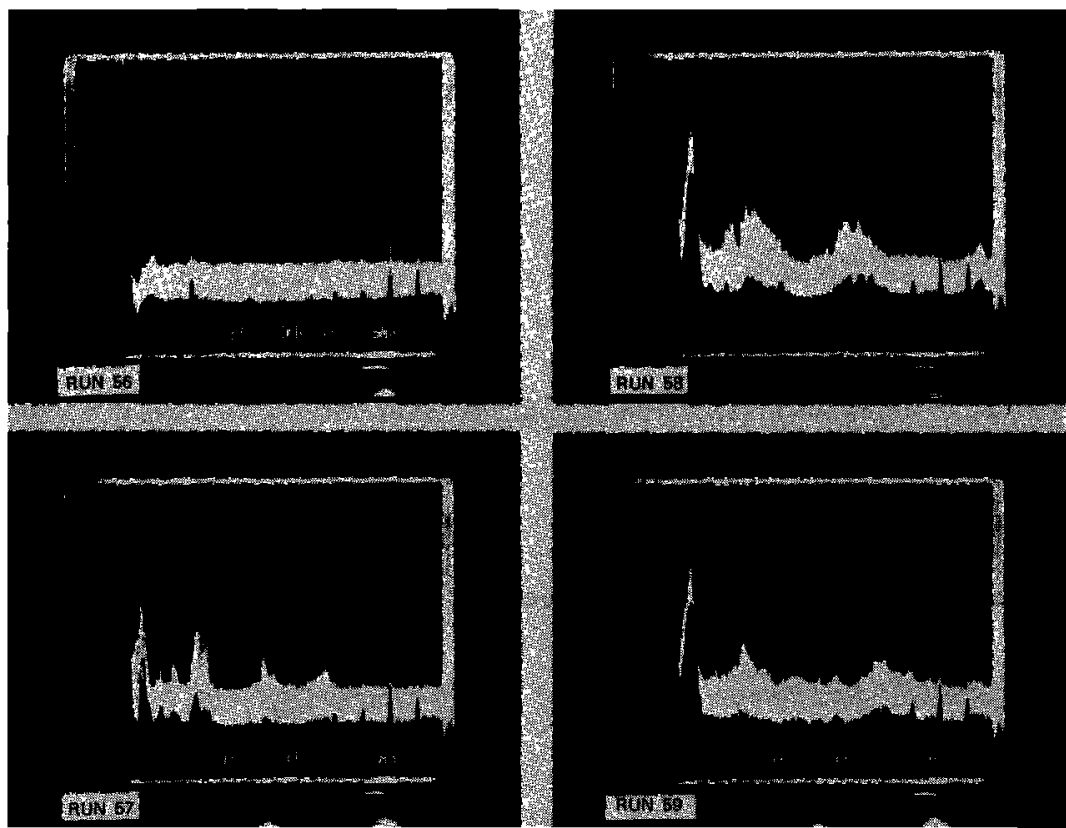

Fig. $5 b$

Fig. 5. (a) Sequence of relative plasma density profiles and (b) associated plasma wave signatures for increasing values of beam current $I_{B}$ at a fixed condition $\left(V_{B}, B\right)=(1.3 \mathrm{keV}, 0.9 \mathrm{G})$ encompassing runs 56-59 (pre-BPD through solid BPD). 

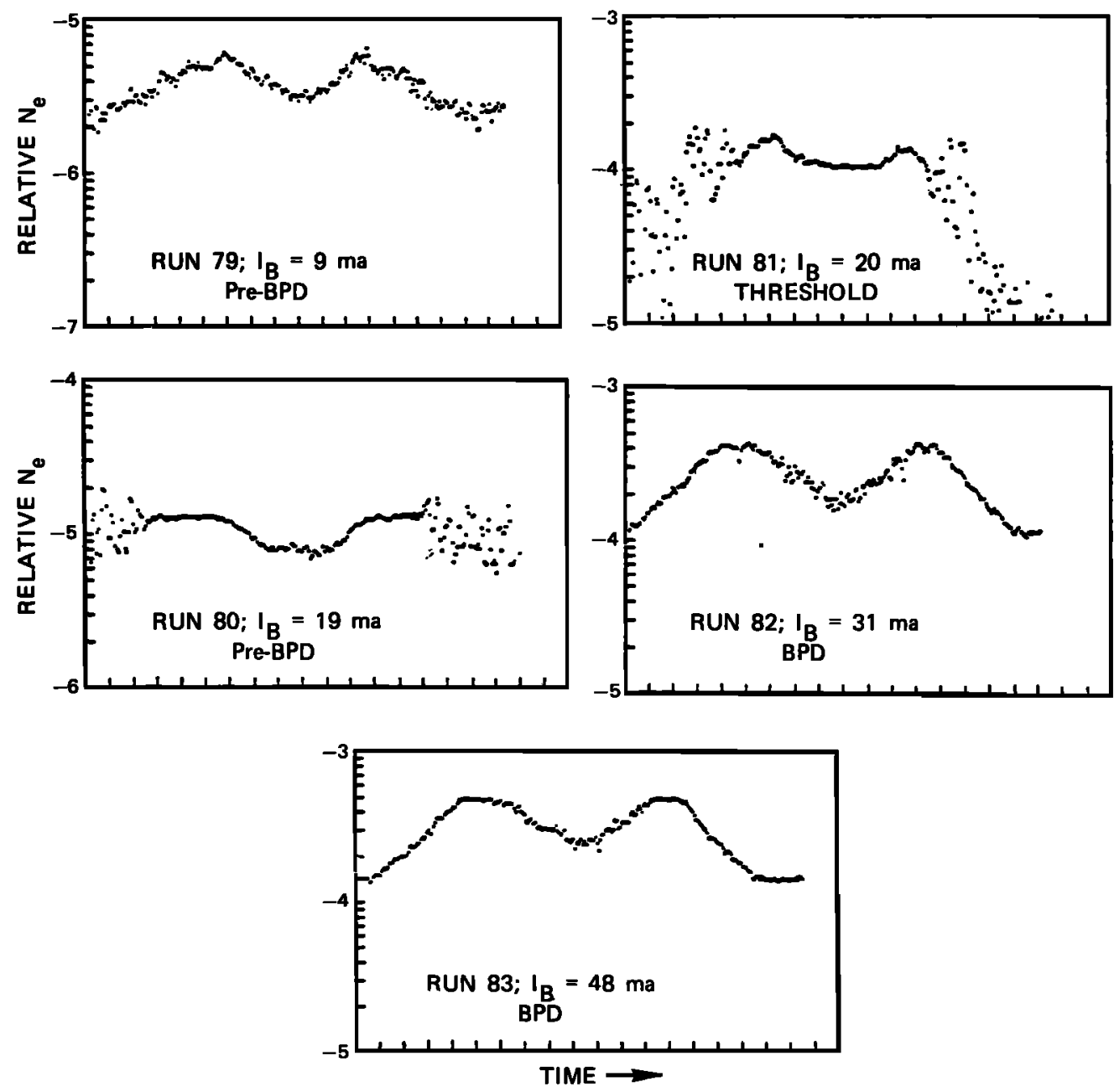

Fig. $6 a$

Fig. 6. (a) Sequence of relative plasma density profiles and (b) associated plasma wave signatures for increasing values of beam current $I_{B}$ at a fixed condition $\left(V_{B}, B\right)=(2.0 \mathrm{keV}, 1.5 \mathrm{G})$ encompassing runs 79 through 83 (pre-BPD through solid BPD).

electron plasma frequency $\omega_{p}$ and the wave energy $W /\left(n_{e} T_{e}\right)$ is greater than $k^{2} \lambda_{D}{ }^{2}$, where $W$ is the field energy density, $n_{e}$ is the ambient electron density, $T_{e}$ is the electron temperature, $k$ is the wave number of the instability, and $\lambda_{D}$ is the Debye length. In order for substantial $W /\left(n T_{e}\right)$ to be built in a finite length system, the instability should be almost absolute. The dispersion relation for a beam plasma system is [Linson and Papadopoulos, 1980; Rowland et al., 1981]

$$
\begin{aligned}
k_{z}^{2}(1 & \left.-\frac{\omega_{p}^{2}}{\omega^{2}}\right)+k_{\perp}^{2}\left(1-\frac{\omega_{p}^{2}}{\omega^{2}-\omega_{c}{ }^{2}}\right) \\
& -k_{z}^{2} \frac{\alpha \omega_{p}^{2}}{\left(\omega-k_{z} v_{b}\right)^{2}}-k_{\perp}^{2} \frac{\alpha \omega_{p}^{2}}{\left(\omega-k_{z} v_{b}\right)^{2}-\omega_{c}{ }^{2}}=0
\end{aligned}
$$

where $k_{\perp}{ }^{2} \equiv \Gamma_{s 1}{ }^{2} / r_{0}{ }^{2}$ with $\Gamma_{s 1}$ the roots of the Bessel function $J_{1}\left(\Gamma_{s 1}\right)=0$ and $r_{0}$ the beam radius. In (5), $\alpha$ is the beam-toplasma density ratio, $\omega_{p}$ and $\omega_{c}$ are the plasma and electron cyclotron frequencies, $v_{b}$ is the beam velocity, and $k_{z}$ is the parallel wave number. On the basis of (5), we can find [Rowland et al., 1981] that an almost absolute instability can be excited near $\omega_{p}$ for

$$
\frac{k_{\perp}^{2}}{k_{z}^{2}} \leq 1
$$

Coupling this inequality with $k_{z} \approx \omega_{p} / v_{b}$ and $k_{\perp}=2.4 / r_{0}$ gives our required threshold condition as

$$
\omega_{p}^{2} \geq \frac{(2.4)^{2} v_{b}^{2} \cos ^{2} \theta_{i}}{r_{0}^{2}}
$$

The criterion (7), with $\theta_{i}$ being the beam's angle of injection relative to the magnetic field, can be generalized to the case where the plasma radius $R$ is different from the beam radius $r_{0}$ [Rowland et al., 1981]. In this case, (7) becomes

$$
\omega_{p}^{2} \geq 2 \frac{v_{b}^{2} \cos ^{2} \theta_{i}}{r_{0}^{2} \ln \left(R / r_{0}\right)}
$$

The value of $r_{0}$ is given by considering both the Larmor radius as well as the term including space charge beam 

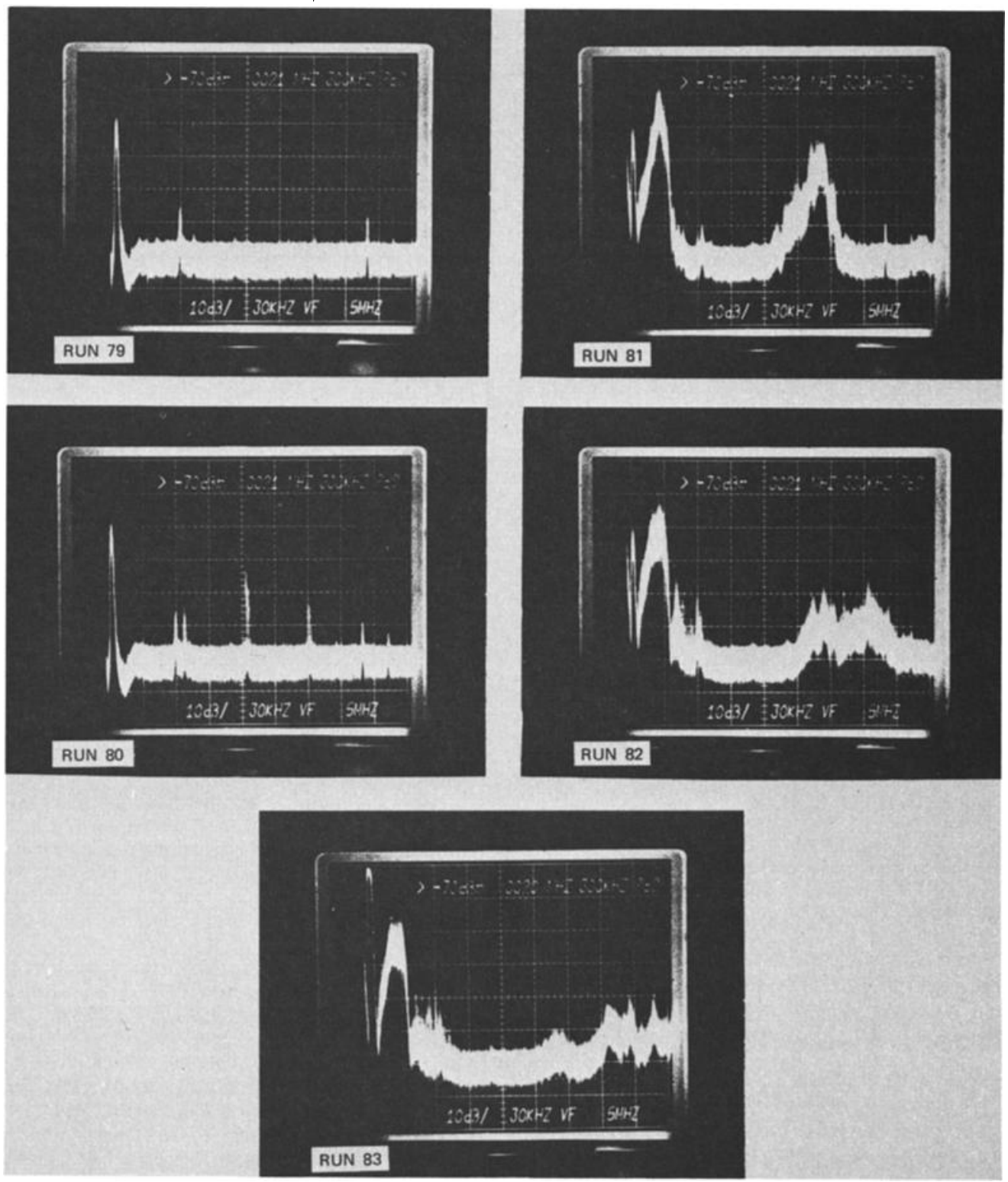

Fig. $6 b$

TABLE 1. Abbreviated Summary of Beam-Plasma Survey

\begin{tabular}{|c|c|cc|c|c|c|c|c|c|}
\hline \multirow{2}{*}{ RUN \# } & \multirow{2}{*}{$\begin{array}{c}\text { BEAM-PLASMA } \\
\text { STATE }\end{array}$} & ELECTRON GUN & \multicolumn{3}{|c|}{ CHAMBER CONDITION } & \multirow{2}{*}{$N_{e} \max$} & \multirow{2}{*}{$f_{c}$} & $f_{p} / f_{c}$ \\
\cline { 3 - 10 } & & $l_{B}$ [ma] & $V_{B}$ [v] & $B$ [g] & $P$ [Torr] & THRUSTER & & & \\
\hline 40 & THRESHOLD & 37 & $1.9\left(10^{3)}\right.$ & 0.9 & $0.7-1.5\left(10^{-5}\right)$ & ON & $3.6\left(10^{6}\right)$ & $2.5\left(10^{6}\right)$ & 6.8 \\
41 & BPD & 47 & $1.9\left(10^{3)}\right.$ & 0.9 & $0.7-1.5\left(10^{-5}\right)$ & ON & $5.6\left(10^{6}\right)$ & $2.5\left(10^{6}\right)$ & 8.4 \\
48 & THRESHOLD & 34 & $1.9\left(10^{3}\right)$ & 0.9 & $0.7-1.5\left(10^{-5}\right)$ & OFF & $3.3\left(10^{6}\right)$ & $2.5\left(10^{6}\right)$ & 6.5 \\
49 & BPD & 45 & $1.9\left(10^{3}\right)$ & 0.9 & $0.7-1.5\left(10^{-5}\right)$ & OFF & $5.0\left(10^{6}\right)$ & $2.5\left(10^{6}\right)$ & 8.0 \\
\hline 57 & THRESHOLD & 18.5 & $1.3\left(10^{3}\right)$ & 0.9 & $0.7-1.5\left(10^{-5}\right)$ & OFF & $1.5\left(10^{6}\right)$ & $2.5\left(10^{6}\right)$ & 4.4 \\
58 & BPD & 28 & $1.3\left(10^{3}\right)$ & 0.9 & $0.7-1.5\left(10^{-5}\right)$ & OFF & $4.5\left(10^{6}\right)$ & $2.5\left(10^{6}\right)$ & 7.6 \\
\hline 63 & THRESHOLD & 7.8 & 800 & 0.9 & $0.84-1.5\left(10^{-5}\right)$ & OFF & $0.98\left(10^{6}\right)$ & $2.5\left(10^{6}\right)$ & 3.5 \\
64 & BPD & 9.9 & 800 & 0.9 & $0.84-1.5\left(10^{-5}\right)$ & OFF & $2.6\left(10^{6}\right)$ & $2.5\left(10^{6}\right)$ & 5.7 \\
69 & THRESHOLD & 7.8 & 800 & 0.9 & $0.7\left(10^{-5}\right)$ & ON & $3.8\left(10^{6}\right)$ & $2.5\left(10^{6}\right)$ & 6.9 \\
70 & BPD & 10 & 800 & 0.9 & $0.7\left(10^{-5}\right)$ & ON & $3.6\left(10^{6}\right)$ & $2.5\left(10^{6}\right)$ & 6.8 \\
\hline 81 & THRESHOLD & 20 & $2.0\left(10^{3}\right)$ & 1.5 & $0.6-1.2\left(10^{-5}\right)$ & OFF & $7.0\left(10^{6}\right)$ & $4.2\left(10^{6}\right)$ & 5.6 \\
82 & BPD & 30.5 & $2.0\left(10^{3}\right)$ & 1.5 & $0.6-1.2\left(10^{-5}\right)$ & OFF & $1.8\left(10^{7}\right)$ & $4.2\left(10^{6}\right)$ & 9.1 \\
86 & THRESHOLD & 12 & $1.3\left(10^{3}\right)$ & 1.5 & $0.6-1.2\left(10^{-5}\right)$ & OFF & $3.9\left(10^{6}\right)$ & $4.2\left(10^{6}\right)$ & 4.2 \\
87 & BPD & 18 & $1.3\left(10^{3}\right)$ & 1.5 & $0.6-1.2\left(10^{-5}\right)$ & OFF & $1.1\left(10^{7}\right)$ & $4.2\left(10^{6}\right)$ & 7.1 \\
\hline
\end{tabular}


TABLE 2. Comparison Between Theory and Experiment

\begin{tabular}{ccccc}
\hline $\begin{array}{c}\text { Run } \\
\text { Number }\end{array}$ & $V_{B}, \mathrm{keV}$ & $I_{B}, \mathrm{ma}$ & $\begin{array}{c}\omega_{p} / \omega_{c} \\
\text { (Calculated) }\end{array}$ & $\begin{array}{c}\omega_{p} / \omega_{c} \\
\text { (Observed) }\end{array}$ \\
\hline 40 & 1.9 & 37 & 4.2 & 6.8 \\
48 & 1.9 & 34 & 4.4 & 6.5 \\
57 & 1.3 & 18.5 & 4.5 & 4.4 \\
63 & 0.8 & 7.8 & 4.7 & 3.5 \\
69 & 0.8 & 6.2 & 5.37 & 6.9 \\
81 & 2 & 20 & 5.95 & 5.6 \\
86 & 1.3 & 12 & 5.55 & 4.2 \\
\hline
\end{tabular}

expansion [Linson and Papadopoulos, 1980]. This gives

$$
r_{0}=2.1 \frac{I_{B}{ }^{1 / 2}}{B V_{B}{ }^{1 / 4}}\left(\ln \frac{r_{0}}{r_{g}}+\frac{33}{\kappa} \sin ^{2} \theta_{d}\right)^{1 / 2} m
$$

where $\kappa$ is gun perveance in micropervs, $r_{g}$ is the gun radius, $I_{B}$ and $V_{B}$ are the beam current in amps and energy in $\mathrm{keV}$, the magnetic field $B$ is in gauss, and $\theta_{d}$ is the beam divergence angle. (We note that equation (8) does not include the possibility of beam neutralization by ambient plasma. This could result in an overestimate of $r_{0}$.) From (8) and (9) and by using $r_{g} \approx 2 \mathrm{~mm}$ and $r_{0} / R \approx \frac{1}{2}$ as observed, we find

$$
\frac{\omega_{p}}{\omega_{c}} \geq \frac{0.5 V_{B}^{3 / 4}}{I_{B}^{1 / 2}} \frac{\cos \theta_{i}}{\left(1+(8.5 / \kappa) \sin ^{2} \theta_{d}\right)^{1 / 2}}
$$

We can see that the $\omega_{p} / \omega_{c}$ ratio at threshold (with $\kappa \approx 1$ microperv) is rather insensitive to the beam injection angle for $\theta_{i} \leqslant 60^{\circ}$. In Table 2 we present the values of $\omega_{p} / \omega_{c}$ computed on the basis of equation (10) by taking $\cos \theta_{i} /(1+$ $\left.(8.5 / \kappa) \sin ^{2} \theta_{d}\right)^{1 / 2} \approx 1$ for near parallel injection. From Table 2 we find the average computed value to be $\omega_{p} / \omega_{c}=4.95$. This result, while subject to moderate uncertainties in $r_{0} / R$, is taken to be in excellent agreement with the experimentally derived conditions (Table 1 and equation (4)) providing complementary arguments that confirm the original suggestion that the ignition of the BPD was coupled to a densitydependent threshold criterion. We should mention that the above theory is consistent with the observational facts that the temperature of the plasma electrons is much lower than the ionization potential [Szuszczewicz et al., 1979], and energetic tails are observed within the beam-plasma core [Szuszczewicz, 1982].

Acknowledgments. The various elements in this investigation were supported in whole or in part by NASA grant NAGW-69, Rice University subcontract NAS 8-33777-1, and NASA/NOAA contract NA79RAE0039. Supplementary funding was also provided by the Office of Naval Research under Program Element 61153N-33 in Task Area RR033-02. We wish to thank J. C. Holmes for his critical care in electronics design of the $\mathrm{P}^{3}$ instrumentation, L. Kegley for technical assistance in experiment execution, and $L$. Linson for his review and comments on the manuscript.

The Editor thanks R. J. Jost and P. J. Kellogg for their assistance in evaluating this paper.

\section{REFERENCES}

Bernstein, W., H. Leinbach, P. Kellogg, S. Monson, T. Hallinan, O. K. Garriott, A. Konradi, J. McCoy, P. Daly, B. Baker, and H. R. Anderson, Electron beam injection experiments: The beam-plas- ma discharge at low pressures and magnetic field strengths, Geophys. Res. Lett., 5, 127, 1978.

Bernstein, W., H. Leinbach, P. J. Kellogg, S. J. Monson, and T. Hallinan, Further laboratory measurements of the beam-plasma discharge, J. Geophys. Res., 84, 7271, 1979.

Bernstein, W., B. A. Whalen, F. R. Harris, A. G. McNamara, and A. Konradi, Laboratory studies of the charge neutralization of a rocket payload during electron beam emission, Geophys. Res. Lett., 7, 93, 1980.

Cambou, F., V. S. Dokoukine, V. N. Ivchenko, G. G. Managadze, V. V. Migulin, O. K. Nazarenko, A. T. Nesmyanovich, A. Kh. Pyatsi, R. Z. Sagdeev, and I. A. Zhulin, The Zarnitza rocket experiment on electron injection, Space Res., 15, 491-500, 1975.

Cambou, F., J. Lavergnat, V. V. Migulin, A. I. Morozov, B. E. Paton, R. Pellat, A. Pvatsi, H. Reme, R. Z. Sagdeev, W. R. Sheldon, and I. A. Zhulin, ARAKS-controlled or puzzling experiment?, Nature, 271, 723, 1978.

Chen, F. F., Plasma Diagnostic Techniques, chap. 4, edited by $\mathbf{R}$. H. Huddlestone and S. L. Leonard, Academic, New York, 1965.

Galeev, A. A., E. V. Mishin, R. Z. Sagdeev, V. D. Shapiro, and V. I. Shevchenko, Discharge in the region around a rocket following the injection of electron beams in the ionosphere, Sov. Phys. Dokl., 21, 641, 1976.

Getty, W. D., and L. D. Smullin, Beam-plasma discharge: Buildup of oscillations, J. Appl. Phys., 34, 3421, 1963.

Hendrickson, R. A., and J. R. Winckler, Echo III: The study of electric and magnetic fields with conjugate echoes from artificial electron beams injected into the auroral zone ionosphere, Geophys. Res. Lett., 3, 409, 1976.

Hess, W. N., M. C. Trichel, T. N. Davis, W. C. Beggs, G. E. Kraft, E. Strasinopoulos, and E. J. R. Maier, Artificial aurora experiment: Experiment and principal results, J. Geophys. Res., 76, $6067,1971$.

Holmes, J. C., and E. P. Szuszczewicz, A versatile plasma probe, Rev. Sci. Instrum., 46, 592, 1975.

Holmes, J. C., and E. P. Szuszczewicz, A plasma probe system with automatic sweep adjustment, Rev. Sci. Instrum., 52, 377, 1981.

Jost, R. J., H. R. Anderson, and J. O. McGarity, Measured electron energy distributions during electron beam-plasma interactions, Geophys. Res. Lett., 7, 509, 1980.

Linson, L. M., and K. Papadopoulos, Review of the status of theory and experiment for injection of energetic electron beams in space, Rep. LAPS-69/SAI-D23-459-LJ, Sci. Appl., Inc., LaJolla, Calif., April 1980.

Monson, S. J., and P. J. Kellogg, Ground observations of waves at $2.96 \mathrm{MHz}$ generated by an 8- to 40-KEV electron beam in the ionosphere, J. Geophys. Res., 83, 121, 1978.

Papadopoulos, K., Theory of beam plasma discharge, in Proceedings of NATO Advanced Research Institute on Artificial Particle Beams in Space Plasma Physics, Plenum, New York, in press, 1982.

Papadopoulos, K., and T. Coffey, Nonthermal features of the auroral plasma due to precipitating electrons, J. Geophys. Res., $79,674,1974 a$.

Papadopoulos, K., and T. Coffey, Anomalous resistivity in the auroral plasma, J. Geophys. Res., 79, 1558, $1974 b$.

Papadopoulos, K., and H. L. Rowland, Collisionless effects on the spectrum of secondary auroral electrons at low altitudes, $J$. Geophys. Res., 83, 5768, 1978.

Rowland, H. L., C. L. Chang, and K. Papadopoulos, Scaling of the beam-plasma discharge, J. Geophys. Res., 86, 9215, 1981.

Szuszczewicz, E. P., Direct measurements of plasma characteristics in space-simulation beam-plasma interactions, paper presented at 20th Aerospace Sciences Meeting, Am. Inst. of Aeronaut. and Astronaut, Orlando, Fla., Jan. 1982.

Szuszczewicz, E. P., and J. C. Holmes, Surface contamination of active electrodes in plasmas: Distortion of conventional Langmuir probe measurements, J. Appl. Phys., 46, 5134, 1975.

Szuszczewicz, E. P., and J. C. Holmes, Reentry plasma diagnostics with a pulsed plasma probe, paper presented at 9th Fluid and Plasma Dynamics Conference, Am. Inst. of Aeronaut. and Astronaut., San Diego, Calif., July 1976.

Szuszczewicz, E. P., and J. C. Holmes, Observations of electron temperature gradients in mid-latitude $E_{s}$ layers, J. Geophys. Res., 82, 5073, 1977.

Szuszczewicz, E. P., D. N. Walker, and H. Leinbach, Plasma 
diffusion in a space simulation beam-plasma discharge, Geophys. Res. Lett., 6, 201, 1979.

Winckler, J. R., The application of artificial electron beams to magnetospheric research, Rev. Geophys. Space Phys., 18, 659, 1980.

Winckler, J. R., R. L. Arnoldy, and R. A. Hendrickson, Echo 2: A study of electron beams injected into the high-latitude ionosphere from a large sounding rocket, J. Geophys. Res., 80, 2083, 1975.

(Received June 29, 1981;

revised October 2, 1981;

accepted October 30, 1981.) 